\title{
Prevalência do aleitamento materno na região noroeste de Campinas, São Paulo, Brasil, 2001
}

\section{Breast-feeding prevalence, northwest region of Campinas, São Paulo, Brazil, 2001}

Daniel Felipe Alves CECCHETTI

Erly Catarina MOURA²

\section{RE S U M O}

\section{Objetivo}

Calcular a prevalência do aleitamento materno entre crianças menores de dois anos de idade, residentes na região Noroeste de Campinas, São Paulo.

\section{Métodos}

Estudo transversal foi conduzido, em 2001, junto a 4103 crianças, questionando sobre data de nascimento, sexo, alimentação e serviço de saúde utilizado. O questionário foi aplicado em 42 postos de vacinação durante a Campanha Nacional de Vacinação contra Poliomielite (Campólio). A dieta foi classificada em amamentação exclusiva, predominante, continuada e aleitamento artificial.

\section{Resultados}

No primeiro semestre, a prevalência de aleitamento materno exclusivo foi de $31,6 \%$ e a de aleitamento total $74,5 \%$. Das crianças com idade entre 6 e 12 meses, 38,0\% recebiam leite materno. No segundo ano, a prevalência de aleitamento materno foi reduzida para $22,1 \%$. O aleitamento materno exclusivo passou de $72,2 \%$ aos 7 dias de idade para 53,8\% aos 15 dias, 33,3\% aos 3 meses, 10,0\% aos 4 meses e 5,7\% aos 6 meses. A prevalência do aleitamento materno total foi de $100,0 \%$ aos 7 dias; $79,1 \%$ aos 3 meses; $54,3 \%$ aos $6 ; 34,4 \%$ aos 12 meses; $26,1 \%$ aos 18 e zero aos 24 meses. A mediana de amamentação exclusiva foi de 67 dias e a de amamentação total foi de 6,6 meses. Das crianças vinculadas ao Sistema Único de Saúde, 42,2\% receberam aleitamento materno, em contraste com $34,4 \%$ das usuárias de serviços privados $(p<0,00001)$. Após controle pela idade, o efeito protetor do Sistema Único de Saúde se manteve, só desaparecendo quando se incluiam as variáveis renda familiar e escolaridade materna.

\footnotetext{
1 Acadêmico, Curso de Medicina, Faculdade de Medicina, Centro de Ciências da Vida, Pontifícia Universidade Católica de Campinas. Bolsista de Iniciação Científica, PIBIC-CNPq.

2 Núcleo de Pesquisas Epidemiológicas em Nutrição e Saúde, Faculdade de Saúde Pública, Universidade de São Paulo. Avenida Dr. Arnaldo, 715, 01246-904, São Paulo, SP, Brasil. Correspondência para/Correspondence to: E.C. MOURA. E-mail: <erlycm@usp.br>.
} 


\section{Conclusão}

A prevalência de aleitamento materno está abaixo das recomendações oficiais.

Termos de indexação: amamentação, bem-estar da criança, epidemiologia, serviços de saúde, saúde pública.

\section{A B S T R A C T}

\section{Objective}

To calculate the prevalence of breast-feeding among children less than two years old, in the northwest region of the city of Campinas, São Paulo, Brazil.

\section{Methods}

A survey was conducted in 2001, with 4103 children, querying about birth date, gender, feeding practices and health services routinely used. The questionnaire was applied at the 42 public immunization centers during the 2001 National Campaign against Polyomielites. The child's diet was categorized as exclusive breastfeeding, predominant breast-feeding, continued breast-feeding and bottle-feeding.

\section{Results}

The prevalences of the exclusive and the total breast-feeding in the first semester were, respectively, 31.6\%, and $74.5 \%$. Among children aged from six to twelve months, 38.0\% were breast-fed. In the second year, the prevalence of breast-feeding was reduced to $22.1 \%$. Exclusive breast-feeding changed from $72.2 \%$ at the $7^{\text {th }}$ day of life, to $53.8 \%$ at the $15^{\text {th }}$ day, to $33.3 \%$ at the $3^{\text {rd }}$ month, to $10.0 \%$ at the $4^{\text {th }}$, and to $5.7 \%$ at the $6^{\text {th }}$ month. The total breast-feeding prevalence was $100.0 \%$ at the $7^{\text {th }}$ day, $79.1 \%$ at the $3^{\text {rd }}$ month, $54.3 \%$ at the $6^{\text {th }}, 34.4 \%$ at the $12^{\text {th }}, 26.1 \%$ at the $18^{\text {th }}$, and none at the $24^{\text {th }}$ month. Exclusive and the total breast-feeding medians were, respectively, 67 days and 6.6 months. Among children who attended the public health system, $42.2 \%$ were breast-fed, against $34.4 \%$ of those who attended a private service $(p<0.00001)$. The protecting effect of the Public Health Service remained, when the statistic model was adjusted for age; conversely, it vanished, when variables like income and maternal education were included in the model.

\section{Conclusion}

The prevalence of breast-feeding is below official recommendations.

Indexing terms: breast feeding, child welfare, epidemiology, health services, public health.

\section{N T R O D U Ç Ã O}

Muito se tem pesquisado acerca dos benefícios trazidos pelo aleitamento materno. Desde meados dos anos 70 até os dias atuais houve grande ampliação do conhecimento científico desta prática, constatando-se a importância do aleitamento materno como meio de proteção contra doenças como alergias, diarréias, doenças crônicas não transmissíveis, doenças respiratórias e, inclusive, malformação da arcada dentária ${ }^{1-5}$. Além de todos esses benefícios, o aleitamento materno fornece os nutrientes essenciais ao crescimento e desenvolvimento do lactente e garante maior aproximação na relação mãe-filho.

$\mathrm{O}$ aleitamento materno traz benefícios a todos os recém nascidos, não importando sua raça, condição social ou econômica. Todavia, no Brasil, país no qual se observam enormes discrepâncias sociais, o aleitamento materno surge como um elemento importante, não só do ponto de vista nutricional, mas também do econômico.

Apesar da amamentação configurar-se como uma importante ferramenta, tanto para a atenuação da difícil realidade da população menos favorecida, quanto para a diminuição da morbidade de crianças com melhores condições sociais, o que se observa são prevalências de aleitamento materno, especialmente, do aleitamento exclusivo, muito aquém do recomendado. Nos anos 70, ponto máximo do declínio da prática da amamentação, houve piora das condições de saúde materno-infantil. Como conseqüência, campanhas foram organizadas e 
o aleitamento materno teve importante incremento na década subseqüente ${ }^{6}$. Todavia, estudos comparativos de épocas mais recentes com a década de 80, relatam manutenção da prevalência de amamentação, como no trabalho desenvolvido em Porto Alegre, RS, no qual, analisou-se e comparou-se a situação da amamentação em 1987 e 1994, obtendo-se freqüência de amamentação no quarto mês de vida igual a $63,9 \%$ e $61,0 \%$, respectivamente e, aos seis meses, $48,5 \%$ e $48,2 \%{ }^{7}$.

No Brasil, de forma geral, houve melhora importante nos índices de aleitamento materno na década de 80, com ritmo mais lento após este período, chegando à estabilização em certos locais, o que mostra a falta de uniformidade com importantes variações no território nacional ${ }^{8}$. Por isso, é de fundamental importância o conhecimento das tendências de amamentação em cada localidade.

Neste sentido, este trabalho tem como objetivo identificar a ocorrência do aleitamento materno para crianças menores de dois anos de idade, residentes no Distrito Noroeste de Campinas, estado de São Paulo, a fim de subsidiar o planejamento de promoções pró-amamentação, além de contribuir com dados epidemiológicos e monitorar os indicadores locais.

\section{M É T O D O S}

Campinas localiza-se a 100 quilômetros ao noroeste da cidade de São Paulo, contando com cerca de 970 mil habitantes. Para fins administrativos e assistenciais, divide-se em cinco grandes regiões: Norte, Sul, Leste, Sudoeste e Noroeste. A região Noroeste é uma das mais densamente povoadas, com aproximadamente 152 mil habitantes. Esta pesquisa, de corte transversal, teve sua população de estudo constituida de crianças menores de 2 anos de idade da região Noroeste de Campinas. Como estratégia amostral, buscou-se entrevistar todas as crianças que compareceram aos 42 postos de vacinação no dia 9 de junho de 2001, durante a primeira fase da Campólio. De um total de 4424 que compareceram à vacinação, 4140 responsáveis por crianças menores de 2 anos de idade foram abordados, sendo que 2 pessoas se negaram a participar do estudo, 19 desconheciam o serviço de saúde mais utilizado pela criança para a puericultura, 3 desconheciam a data exata do nascimento da criança e 23 não conheciam detalhes sobre sua alimentação. Estes casos foram excluídos da análise, restando 4093 casos válidos.

Para a coleta de dados, utilizou-se o método do status-quo ou current status por sua praticidade, baixo custo e confiabilidade, além de permitir o cálculo de medianas em estudos transversais ${ }^{6}$. Os responsáveis pelas crianças foram questionados sobre: grau de parentesco com a criança, data de nascimento, sexo, alimentos ingeridos nas últimas 24 horas e serviço de saúde mais usado pela criança. As entrevistas foram conduzidas por estudantes dos cursos de Nutrição e Medicina da Pontifícia Universidade Católica de Campinas (PUC-Campinas), os quais foram especialmente treinados e supervisionados.

A dieta recebida pelas crianças foi classificada conforme recomendação da Organização Mundial da Saúde9, em amamentação exclusiva (só leite materno), amamentação predominante (leite materno associado a outro líquido, exceto fórmula e/ou leite de vaca), amamentação continuada (leite materno associado a outro alimento, inclusive fórmula e/ou leite de vaca) e aleitamento artificial (fórmula e/ou leite de vaca associado a outro alimento, exceto leite materno). Considerou-se aleitamento total quando a criança recebia leite materno de forma exclusiva ou não. Foram calculadas: a idade da criança no dia da vacinação, a prevalência do aleitamento materno exclusivo e total, além das medianas de aleitamento materno exclusivo e total. A associação entre a presença de amamentação conforme o sexo e o serviço de saúde mais usado (serviço privado ou Serviço Unificado de Saúde-SUS) foi investigada pelo teste do qui-quadrado, considerando-se nível de significância de 5\%. Para o cálculo de risco de desmame, 
empregou-se o odds-ratio e nível de confiança de 95\%. Regressão logística foi executada, após controle para a idade da criança, para verificar a influência do serviço de saúde na amamentação. Os dados foram processados no Statistical Package for Social Sciences.

O estudo contou com a aprovação do Comitê de Ética em Pesquisa em Seres Humanos da Faculdade de Ciências Médicas da Pontifícia Universidade Católica de Campinas.

\section{RES ULTA D O S}

Foram abordados 4103 responsáveis por crianças menores de 2 anos de idade, sendo a maioria mães $(79,2 \%)$, seguidas por pais $(9,7 \%)$, outros familiares $(8,9 \%)$ e amigos $(2,2 \%)$. Das crianças estudadas, $48,9 \%$ eram do sexo feminino e 51,1\% do masculino. A maioria era SUS-dependente $(68,0 \%), 29,9 \%$ utilizavam convênios médicos e $2,1 \%$, atendimento particular. O centro de saúde, onde as crianças receberam a vacina, era utilizado rotineiramente por $48,7 \%$ dos entrevistados, 7,0\% deles utilizavam outros centros de saúde e 12,8\% recorriam ao ambulatório de pediatria do hospital universitário da PUC-Campinas.

Em relação a distribuição das crianças segundo o tipo de amamentação no primeiro semestre de vida (Tabela 1), nota-se um progressivo declínio da amamentação exclusiva com a idade. Entre as crianças menores de 4 meses com aleitamento materno predominante, 50,4\% recebiam água, $44,8 \%$ chá e $4,3 \%$ suco. A fórmula e/ou leite de vaca esteve presente na dieta de $62,1 \%$ das crianças menores de 4 meses com aleitamento complementado. No presente estudo, observou-se, em relação à idade, uma diminuição progressiva do aleitamento materno exclusivo e total. $\mathrm{O}$ aleitamento materno exclusivo passou, de $72,2 \%$ aos 7 dias de idade, para $53,8 \%$ aos 15 dias, para 33,3\% aos 3 meses, $10,0 \%$ aos 4 meses e $5,7 \%$ aos 6 meses. A prevalência do aleitamento materno foi de $100,0 \%$ aos 7 dias, 79,1\% aos 3 meses, 54,3\% aos 6 meses, $34,4 \%$ aos 12 meses, $26,1 \%$ aos 18 meses e zero aos 24 meses. Paralelamente, a presença de fórmula e/ou leite de vaca atingiu, nas mesmas idades, respectivamente 5,6\%, 45,8\%, $68,6 \%, 87,5 \%, 95,7 \%$ e $100,0 \%$ das crianças.

A Tabela 2 mostra redução drástica do aleitamento materno já no segundo semestre de vida, quando a fórmula e/ou leite de vaca esteve presente na dieta de $77,8 \%$ das crianças. Entre as crianças com idade entre 6 e 12 meses, observa-se que apenas 38,0\% recebiam leite materno, sendo que, no segundo ano de vida, a prevalência de aleitamento materno foi reduzida para $22,1 \%$ das crianças.

A mediana de amamentação exclusiva foi de 67 dias, enquanto a mediana de amamentação total foi de 6,6 meses. Não se encontrou diferença significante entre a presença de amamentação conforme o sexo $(p=0,10): 38,4 \%$ dos meninos e $40,9 \%$ das meninas eram amamentados. Todavia, $42,2 \%$ das crianças vinculadas ao SUS recebiam

Tabela 1. Distribuição das crianças menores de 6 meses conforme tipo de amamentação e idade. Distrito de Saúde Noroeste, Campinas, 2001.

\begin{tabular}{|c|c|c|c|c|c|c|c|c|c|c|}
\hline \multirow{3}{*}{ Idade (meses) } & \multicolumn{8}{|c|}{ Tipo de amamentação } & \multirow{2}{*}{\multicolumn{2}{|c|}{ Total }} \\
\hline & \multicolumn{2}{|c|}{ Exclusiva } & \multicolumn{2}{|c|}{ Predominante } & \multicolumn{2}{|c|}{ Complementada } & \multicolumn{2}{|c|}{ Artificial } & & \\
\hline & $\mathrm{n}$ & $\%$ & $\mathrm{n}$ & $\%$ & $\mathrm{n}$ & $\%$ & $\mathrm{n}$ & $\%$ & $\mathrm{n}$ & $\%$ \\
\hline $0 \vdash 1$ & 71 & 60,2 & 40 & 33,9 & 1 & 0,8 & 6 & 5,1 & 118 & 100,0 \\
\hline $1 \vdash 2$ & 93 & 51,1 & 68 & 37,4 & 2 & 1,1 & 19 & 10,4 & 182 & 100,0 \\
\hline $2 \vdash 3$ & 72 & 37,9 & 68 & 35,8 & 6 & 3,2 & 44 & 23,2 & 190 & 100,0 \\
\hline $3 \vdash 4$ & 59 & 30,4 & 56 & 28,9 & 20 & 10,3 & 59 & 30,4 & 194 & 100,0 \\
\hline $4 \vdash 5$ & 24 & 14,6 & 35 & 21,3 & 50 & 30,5 & 55 & 33,5 & 164 & 100,0 \\
\hline $5 \vdash 6$ & 7 & 3,9 & 15 & 8,3 & 79 & 43,9 & 79 & 43,9 & 180 & 100,0 \\
\hline
\end{tabular}


aleitamento materno, contrastando com os $34,4 \%$ apenas, das usuárias de serviços privados $(p<0,00001)$, isto significa que o risco de desmame de uma criança usuária do serviço privado foi $40 \%$ maior do que o de criança SUS-dependente (odds ratio $=1,4$; intervalo de confiança entre 1,2 e 1,6). Não houve diferença significativa quanto à amamentação exclusiva para os menores de 6 meses, que incluiu 31,2\% dos usuários do SUS e 32,9\% dos usuários dos serviços privados. A regressão logística, após ajuste pela idade, mostrou que o efeito protetor do SUS em relação ao aleitamento materno total se manteve $(p<0,0001)$ (Figura 1).

Tabela 2. Distribuição das crianças até 2 anos de idade conforme presença de amamentação e idade. Distrito de Saúde Noroeste, Campinas, 2001.

\begin{tabular}{|c|c|c|c|c|c|c|}
\hline \multirow{3}{*}{ Idade (semestres) } & \multicolumn{4}{|c|}{ Amamentação } & \multirow{2}{*}{\multicolumn{2}{|c|}{ Total }} \\
\hline & \multicolumn{2}{|c|}{ Presente } & \multicolumn{2}{|c|}{ Ausente } & & \\
\hline & $n$ & $\%$ & $n$ & $\%$ & $n$ & $\%$ \\
\hline 1 & 766 & 74,5 & 262 & 25,5 & 1028 & 100,0 \\
\hline 2 & 431 & 38,0 & 702 & 62,0 & 1133 & 100,0 \\
\hline 3 & 286 & 27,0 & 772 & 73,0 & 1058 & 100,0 \\
\hline 4 & 141 & 16,1 & 733 & 83,9 & 874 & 100,0 \\
\hline
\end{tabular}

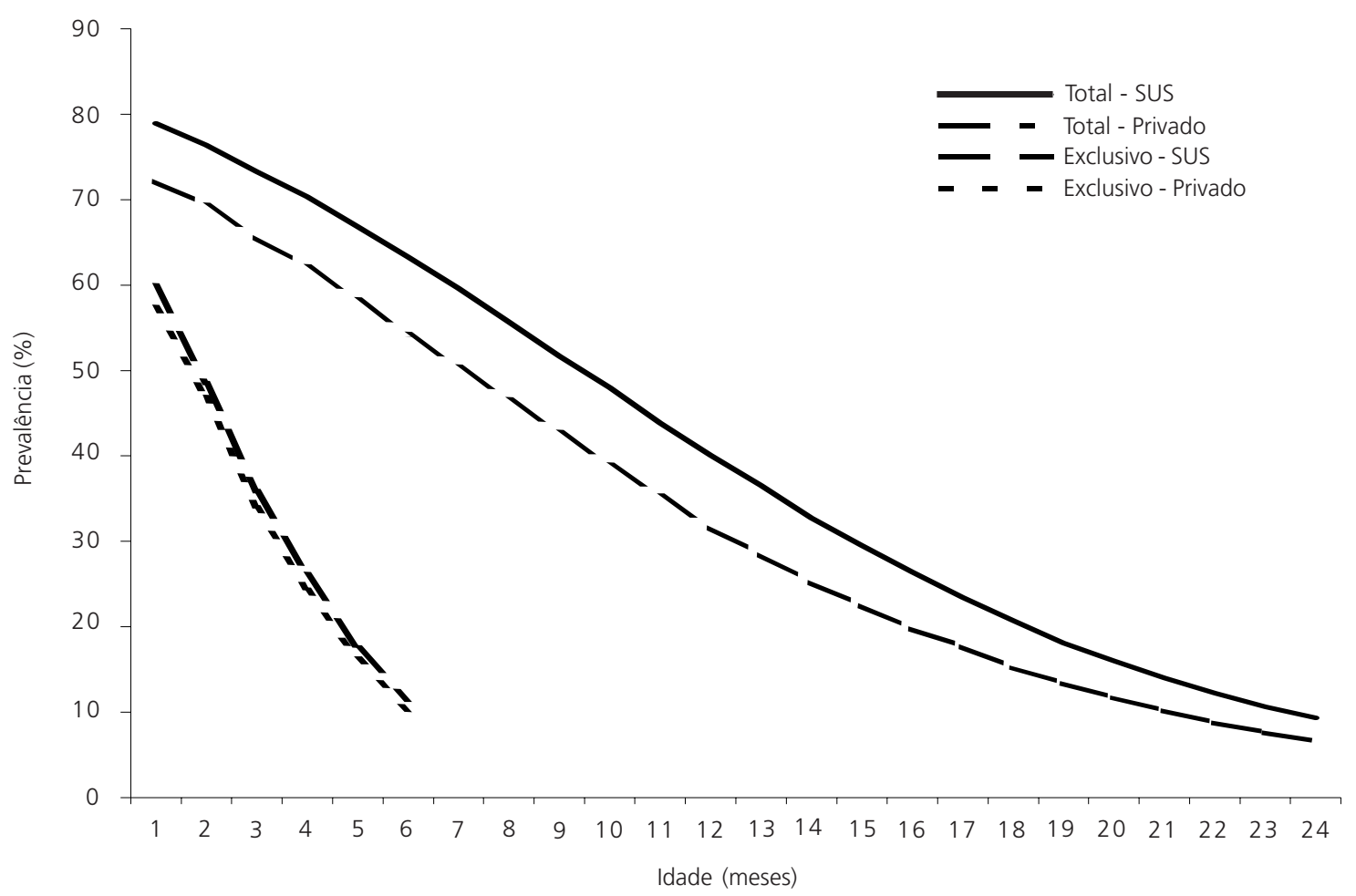

Figura 1. Prevalência de aleitamento materno exclusivo e total e fórmula e/ou leite de vaca conforme a idade. Distrito de Saúde Noroeste, Campinas, 2001. 


\section{I S C US S Ã O}

No presente estudo, identificaram-se $60,2 \%$ das crianças menores de um mês em amamentação exclusiva, cuja prevalência caiu para quase a metade, $30,4 \%$, no quarto mês, chegando a apenas 3,9\% no sexto mês, ficando a mediana em 67 dias. No que se refere à amamentação total, a mediana foi de 6,6 meses, com prevalência de $74,5 \%$ no primeiro semestre de idade, caindo para $38,0 \%$ no segundo e chegando a $16,1 \%$ no quarto semestre. Os dados apontam, ainda, maior prevalência de amamentação total entre as crianças SUS-dependentes, mesmo após ajuste para a idade, não havendo diferença quanto ao sexo. Não se encontrou diferença estatística ao se correlacionarem as variáveis sexo e serviços de saúde, no que se refere ao aleitamento materno exclusivo.

O estudo tipo status-quo, utilizado nesta investigação, tem a vantagem da rapidez, possibilitando a aplicação em elevado número de pessoas e compensando a falta de detalhes de informações, que somente seriam obtidas em estudos longitudinais, com amostras populacionais menores e entrevistas mais demoradas. Todavia, deve-se atentar para o cálculo de prevalências por faixa etária ou por determinada idade, para comparações com dados da literatura. Por exemplo: a prevalência de aleitamento materno exclusivo no primeiro mês de vida, isto é entre 1 e 30 dias, foi de $60,2 \%$, enquanto que com 1 mês de idade (28 a 33 dias) foi de 45,9\%.

Outro fator limitante a ser considerado é a garantia das informações. Caso a fonte não seja fidedigna, ela deverá ser desconsiderada. Neste estudo, quase $80 \%$ das informações foram fornecidas pelas mães das crianças, as pessoas mais próximas das crianças na cultura brasileira. Embora os dados incompletos se refiram a 0,6\% das crianças, em apenas $0,1 \%$ dos casos houve comprometimento da identificação do tipo da amamentação recebida.

Os dados obtidos da região Noroeste de Campinas apontam para $95 \%$ das mães amamentando no primeiro mês de vida, o que sugere $100 \%$ das mães virtualmente iniciando a amamentação ao nascimento, valor superior ao obtido em Ouro Preto, MG, em 1996/199710, e similar aos identificados em 1996 em Montes Claros, MG ${ }^{11}$, e em 1998 em Pernambuco ${ }^{12}$.

Comparativamente aos dados registrados na literatura nacional, os obtidos na região Noroeste de Campinas apresentaram prevalência de aleitamento materno exclusivo superior aos obtidos em Botucatu, no estado de São Paulo ${ }^{13}$, em Montes Claros ${ }^{11}$, Ouro Preto ${ }^{10}$ e Belo Horizonte, no estado de Minas Gerais; também prevaleceram sobre os dados de ocorrência registrados no Ceará, no Distrito Federal, Goiás, e em Florianópolis, SC, porém, tal prevalência foi ligeiramente menor que a prevalência encontrada em Feira de Santana, BA, em $1995^{14}$.

A mediana de aleitamento materno exclusivo obtida neste estudo, embora esteja muito aquém da recomendação oficial, foi maior do que as medianas identificadas em Ouro Preto ${ }^{10}$, Montes Claros ${ }^{11}$, Pernambuco ${ }^{12}$, Botucatu ${ }^{13}$, bem como do que a média das diferentes regiões do Brasil e do país como um todo ${ }^{8}$.

A região Noroeste de Campinas também apresentou prevalência de amamentação total, superior a muitas localidades do Brasil, como por exemplo, Ceará e Distrito Federal em 1994, Feira de Santana, BA, em 1995, Goiás ${ }^{14}$, e também superior à média nacional obtida em $1999^{8}$. Todavia, a mediana de amamentação total foi de 6,6 meses, ou seja, 200 dias, valor inferior aos verificados em Montes Claros ${ }^{11}$, no Rio de Janeiro, nas regiões Norte, Nordeste, Centro-Oeste e Sul ${ }^{8}$, contudo, este valor foi similar ao encontrado em Ouro Preto ${ }^{10}$ e superior aos verificados na região Centro-Leste e em São Paulo .

No que se refere à introdução precoce de alimentos, os dados da região Noroeste de Campinas mostram menor ocorrência do que em Botucatu $^{13}$ e em Pernambuco ${ }^{12}$. Na população estudada, a idade mediana para a introdução de chá, foi de 61 dias, valor semelhante ao identificado na região Sul do país e o dobro do encontrado em Ouro Preto e Montes Claros ${ }^{8}$. 
Este estudo referente à população da região Noroeste do município de Campinas, possibilita subsidiar o Distrito de Saúde local para o planejamento em saúde visando à promoção do aleitamento materno. Apesar dos dados encontrados serem privilegiados quando comparados aos dados nacionais, a situação neste município quanto à amamentação exclusiva mantém-se estagnada nos últimos anos. Dados da mesma região, não publicados, mostram mediana de 2,2 meses em 1997 e de 1,9 em 1999, retornando a 2,2 em 2001, embora a mediana de amamentação total tenha passado de 5,0 meses em 1997, para 5,8 em 1999, avançando para 6,6 meses em 2001.

A mediana da introdução de alimentos complementares foi de 61 dias, sendo mais precocemente introduzidos o chá e a fórmula e/ou o leite de vaca (mediana=91,5 dias), levando ao desmame precoce, cuja mediana foi de 122 dias. $O$ presente estudo vem ressaltar a necessidade de promoção do aleitamento materno, não somente para estimular a amamentação, mas também para retardar a introdução de alimentos complementares, a qual leva ao desmame precoce.

Os dados obtidos neste estudo apontam para um efeito protetor do SUS quanto à presença de amamentação total, efeito que se mantém após controle da idade da criança. Todavia, sabe-se que populações mais pobres tendem a amamentar por mais tempo e que a população dependente do SUS é economicamente mais carente. Dados de cerca de $10 \%$ da população estudada, utilizados em outro estudo, mostram que a população SUS-dependente tem menor renda familiar mensal per capita $\mathrm{R} \$ 146,00$ para a população SUS- dependente e $\mathrm{R} \$ 340,00$ para a do serviço de saúde privado $(p<0,001)$, além de menor escolaridade materna: 7,0 2,9 anos de escola, para a mãe SUS-dependente, e 9,9 93,0 anos para a mãe atendida no serviço privado $(p<0,001)$. Embora o tempo de escolaridade não difira com a presença ou ausência da amamentação $(7,8 \pm 3,2$ anos para as mulheres que amamentam e 8,1 13,2 anos) para aquelas que não amamentam, contrariamente aos achados de outros autores $s^{6,7,15}$, a renda difere: $R \$ 183,00$ reais para as famílias cuja criança era amamentada e $\mathrm{R} \$ 242,00$ para as não amamentadas $(p=0,003)$. Incluindo-se as variáveis, renda familiar e anos de estudo materno, no modelo de regressão logística, o efeito do SUS desaparece; apenas a renda se mantém associada com a presença/ausência de amamentação, $p=0,002$. Como o município de Campinas foi um dos pioneiros na implantação da atenção primária, oferecendo atenção integral e contínua à população em geral, em especial à materno-infantil, sugere-se que esse histórico, além da gama de ações educativas ofertadas na rede básica, pode explicar a anulação do efeito da (baixa) escolaridade.

Estes dados remetem à discussão do papel do setor saúde na promoção do aleitamento materno. É importante ressaltar que, entre as crianças atendidas pelo SUS, 42,2\% recebiam leite materno, ao passo que, entre as crianças atendidas no sistema privado, apenas $34,4 \%$ eram amamentadas. Estes dados devem reforçar o compromisso das unidades básicas de saúde com a política nacional de apoio à amamentação, embora esta política este apoio seja ainda incipiente. Deste modo, estudos de prevalência como este são importantes para avaliar o impacto das ações realizadas e redirecionar a atuação de profissionais e serviços, tendo como meta atingir-se, em todas as faixas de renda, a amamentação exclusiva até os seis meses de idade e a complementar até os dois anos ou mais.

\section{REFERÊ NCIAS}

1. Victora CG, Smith PG, Vaughan JP, Nobre LC, Lombardi C, Teixeira AMB, et al. Evidence for protection by breast-feeding against infant deaths from infectious diseases in Brazil. Lancet. 1987; 2:319-22

2. Scariati PD, Grummer-Strawn LM, Fein SB. A longitudinal analysis of infant morbity and the extent of breastfeeding in the United States. Pediatrics. [serial online] 1997; 99:E5. Available from: URL: http://www. pediatrics.org/cgi/content/ full/107/4/e5 
3. Guerra ME. Influencia del amamantamiento en el desarrollo de los maxilares. Acta Odontologica Venezuelana. 1999; 37:6-10.

4. Von Kries R, Koletzko B, Sauerwald T, Von Mutius E. Does breast-feeding protect against childhood obesity? Adv Exp Med Biol. 2000; 478:29-39.

5. Arifeen $S$, Black RE, Antelman G, Baqui A, Caulfield $\mathrm{L}$, Becker $\mathrm{S}$. Exclusive breastfeeding reduces acute respiratory infection and diarrhea deaths among infants in Dhaka Slums. Pediatrics. [serial online] 2001; 108:E67. Available from: URL: http:// www. pediatrics. org/cgi/content/full/107/4/e67

6. Venâncio SI, Monteiro CA. A tendência da prática da amamentação no Brasil nas décadas de 70 e 80. Rev Bras Epidemiol. 1998; 1:40-9.

7. Kummer SC, Giugliani ERJ, Susin LO, Folletto JL, Lermen NR, Wu VYJ, et al. Evolução do padrão de aleitamento materno. Rev Saúde Pública. 2000; 34:143-8.

8. Brasil. Ministério da Saúde. Secretaria de Política de Saúde. Organização Pan Americana de Saúde. Guia alimentar para crianças menores de 2 anos. Brasília: MS; 2002.

9. Organização Mundial da Saúde. Indicadores para avaliar as prácticas de lactância materna. Genebra: OMS; 1992.

10. Passos MC, Lamounier JA, Silva CAM, Freitas SN, Baudson MFR. Práticas de amamentação no município de Ouro Preto, MG. Rev Saúde Pública. 2000; 34:617-22.

11. Caldeira AP, Goulart EMA. 2000. A situação do aleitamento materno em Montes Claros, Minas Gerais: estudo de uma amostra representativa. J Pediatr. 2000; 76:65-72.

12. Marques NM, Lira PIC, Lima MC, Silva NL, Batista Filho $M$, Huttly SRA, et al. Breastfeeding and early weaning practices in Northeast Brazil: A longitudinal study. Pediatrics [serial online]. 2001; 108:E66. Available from: URL: http://www. pediatrics.org/cgi/content/full/107/4/e 66

13. Carvalhaes MABL, Parada CMGL, Manoel CM Venâncio SI. Diagnóstico da situação do aleitamento materno em área urbana do Sudeste do Brasil: utilização de metodologia simplificada. Rev Saúde Pública. 1998; 32:430-6.

14. Araújo MFM. Situação e perspectivas do aleitamento materno no Brasil. In: Carvalho RM, Tamez RN. Amamentação: bases científicas para a prática profissional. Rio de Janeiro: Guanabara Koogan; 2002. p.1-9.

15. Venâncio SI, Escuder MML, Kitoko P, Rea MF, Monteiro CA. Freqüência e determinantes do aleitamento materno em municípios do Estado de São Paulo. Rev Saúde Pública. 2002, 36:313-8.

Recebido para publicação em 17 de junho de 2003 e aceito em 19 de fevereiro de 2004. 